\title{
Observatorio
}

\section{Sanidad digital y gestión del conocimiento: nuevos escenarios asistenciales para un sistema sanitario de excelencia}

\author{
Por David Novillo-Ortiz
}

\begin{abstract}
Resumen: El presente y futuro de la información en salud tienen su base en la sanidad digital y en la gestión del conocimiento. Parte de este futuro se analizó en la Conferencia de Alto Nivel Ministerial sobre Sanidad Digital, celebrada en Barcelona en marzo de 2010, posicionando la sanidad digital como un elemento de integración en las políticas europeas. Este trabajo también aborda la problemática del acceso a información sobre salud en internet y las políticas de gestión del conocimiento como una herramienta eficaz en la mejora de la práctica clínica y la búsqueda de un sistema sanitario de excelencia.
\end{abstract}

Palabras clave: Sanidad digital, Gestión del conocimiento, Información en salud, Internet, Sociedad del conocimiento, Profesional de la información, Tecnología

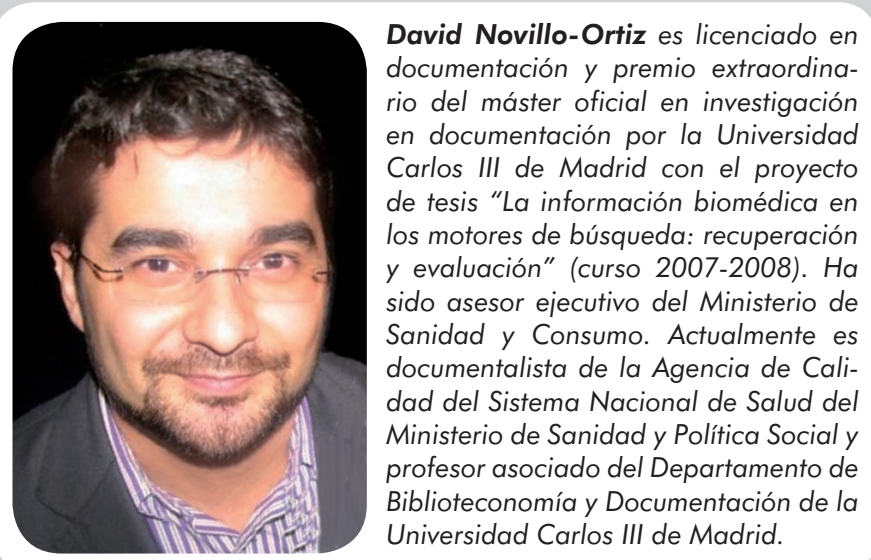

David Novillo-Ortiz es licenciado en documentación y premio extraordinadocumáster oficial en investigación Carlos III de Madrid con el proyecto de tesis "La información biomédica en los motores de búsqueda: recuperación y evaluación" (curso 2007-2008). Ha sido asesor ejecutivo del Ministerio de Sanidad y Consumo. Actualmente es documentalista de la Agencia de Calidad del Sistema Nacional de Salud del Ministerio de Sanidad y Política Social y Pesoriado del Departo de Universidad Carlos III de Madrid.

Title: eHealth and knowledge management: new medical care settings for an excellent health system

Abstract: The present and the future of health information are based on eHealth and knowledge management. Some aspects of this future were discussed at the High Level Ministerial Conference on eHealth, held in Barcelona in March 2010 , where eHealth was seen as a tool for integration in European politics. The problem of access to health information on the internet and knowledge management policies as an effective tool in the improvement of clinical practice and the search for an excellent health system are also analyzed in this article.

Keywords: eHealth, Knowledge management, Health information, Internet, Knowledge society, Information professional, Information technologies, Social web, Web 2.0

Novillo-Ortiz, David. "Sanidad digital y gestión del conocimiento: nuevos escenarios asistenciales para un sistema sanitario de excelencia". El profesional de la información, 2010, mayo-junio, v. 19, n. 3, pp. 225-229.

DOI: 10.3145/epi.2010.may.01

\section{Introducción}

NOS ENCONTRAMOS ANTE UN MUNDO CONECTADO EN REDES en el que la audiencia pasa a ser el contenido. Los pacientes y la ciudadanía requieren la mejor y más fiable información y los profesionales sanitarios utilizan cada vez más las tecnologías de la información y la comunicación (TIC), en particular internet, para compartir sus conocimientos e inquietudes profesionales.

El presente y futuro de la información biosanitaria tienen su base en la sanidad digital o ehealth y en la gestión del conocimiento, como los grandes espacios de interacción sobre los que están surgiendo nuevas oportunidades y nuevos retos en el ámbito sanitario. Es en este escenario en el que los profesionales de la información tienen la oportunidad de liderar, sin complejos, un papel decisivo como intermediarios en la alfabetización digital, en la investigación, y entre los distintos niveles y los diversos actores de la atención sanitaria.

\section{Sanidad digital:}

\section{la tecnología al servicio de la salud}

Podríamos definir la sanidad digital o ehealth como la unión perfecta entre humano-máquina para facilitar y mejorar la calidad de vida de la ciudadanía. 
Las TIC se han convertido en una herramienta habitual en el funcionamiento de cualquier sistema sanitario, tanto en el nivel administrativo (ayudando a diversificar los canales de comunicación) como en el ámbito clínico (generalizando la historia clínica digital o electrónica, implementando la prescripción informatizada de recetas o desarrollando aplicaciones de ayuda al diagnóstico), lo que facilita la relación entre los diferentes profesionales -también para compartir sus experiencias en el aprendizaje ${ }^{1}-\mathrm{y}$ entre éstos y los pacientes a lo largo de todo el proceso asistencial ${ }^{2}$.

En este sentido, el pasado mes de marzo de 2010 se celebró en Barcelona la Conferencia de alto nivel ministerial sobre sanidad digital ${ }^{3}$, que coincidió con la Conferencia y exposición del mundo de las tecnologías de la información sanitaria (World of health it conference and exhibition $)^{4}$. Este evento reunió a la industria nacional e internacional más avanzada del sector. Se dieron cita cerca de 150 ponentes, más de 2.500 delegados, 7.500 seguidores a través de las redes sociales y un centenar de expositores. El principal objetivo fue posicionar la sanidad digital como un elemento de integración en las políticas europeas, contribuyendo así al crecimiento económico y a la innovación tecnológica, al empleo y a la cohesión social y territorial de la Unión, lo que ayudará a mejorar la calidad de vida de los ciudadanos. Prueba de este interés fue la firma de una declaración institucional de cooperación europea en sanidad digital que trata los siguientes cinco puntos: 1) garantizar el compromiso político y estratégico; 2) construir confianza y aceptación; 3) proporcionar claridad legal y ética y garantizar la protección de datos personales médicos; 4) resolver cuestiones relacionadas con la interoperabilidad; y 5) vincular la política de la sanidad digital a las políticas de competitividad, innovación e investigación, así como a las de cohesión e inclusión.

En el ámbito europeo, el alto nivel de desarrollo de este sector en España y la experiencia en la coordinación de las políticas autonómicas de una estructura descentralizada del sistema sanitario han posibilitado que nuestro país lidere un marco común para el desarrollo conjunto y la aplicación de una política de implantación de la gobernanza en sanidad digital en el espacio europeo, redundando en la competitividad de las empresas europeas y españolas del sector de Tecnologías de la Información y la Comunicación en salud. Desde que esta propuesta fue aprobada son ya 25 los países y 7 las asociaciones (profesionales, industria y pacientes) adheridos a esta iniciativa.

En el terreno internacional, otra actividad que creará nuevas oportunidades y desarrollos en el marco de la información biomédica será el Foro global para la innovación en eSalud centrada en las personas, formado por personas expertas de los cinco continentes, y cuyo principal objetivo es fomentar proyectos colaborativos a escala internacional que aceleren la implantación de sistemas de salud basados en las necesidades de los ciudadanos, apoyándose en las tecnologías de la información y la comunicación.

\section{Información, internet y salud}

La dificultad para acceder a información fiable y de calidad sobre salud disponible a través de internet es otro de los desafíos más importantes al que se enfrentan todos los agentes involucrados: ciudadanía, autoridades sanitarias autonómicas y centrales, profesionales sanitarios, industria farmacéutica, las asociaciones y sociedades científicas del sector y, por supuesto, los profesionales de la información.

Desde su aparición, los principales problemas de la Web tienen una difícil solución: por un lado, el exceso de información que sobre cualquier tema podemos encontrar (según la consultora NetCraft $t^{5}$, en marzo de 2010, se tuvo constancia de la existencia de $206 \mathrm{mi}-$ llones de servidores activos en internet); por otro lado, esta situación genera un constante ruido documental que provoca la falta de información específica y de evaluación de la fiabilidad de lo que se está consultando. Este problema se agrava aún más si lo que se intenta es conocer información sobre aspectos relacionados con las ciencias de la salud ${ }^{6}$.

\section{"España lidera un marco común para el desarrollo de una política de implantación de la gobernanza en sanidad digital en el espacio europeo"}

No obstante, se ha demostrado que las oportunidades que nos ofrecen las tecnologías de la información las convierten en un recurso útil, creíble y relevante, que proporciona un impacto en la mejora de la práctica clínica y la calidad asistencial. Internet permite un acceso rápido e inmediato a la información y al conocimiento, siendo un medio disponible en todo momento, que facilita la actualización de la información y la difusión del conocimiento científico frente a los hábitos tradicionales de consulta de información en salud utilizados, como pueden ser la revisión de fuentes en soporte papel o las relaciones entre compañeros ${ }^{7,8}$.

Si hacemos referencia al consumo de información biosanitaria a través de internet, en relación a la búsqueda de información en salud, el número de personas usuarias, según datos del Instituto Nacional de Estadística (INE) y de Eurostat, indica que es alto y está en constante progresión. 


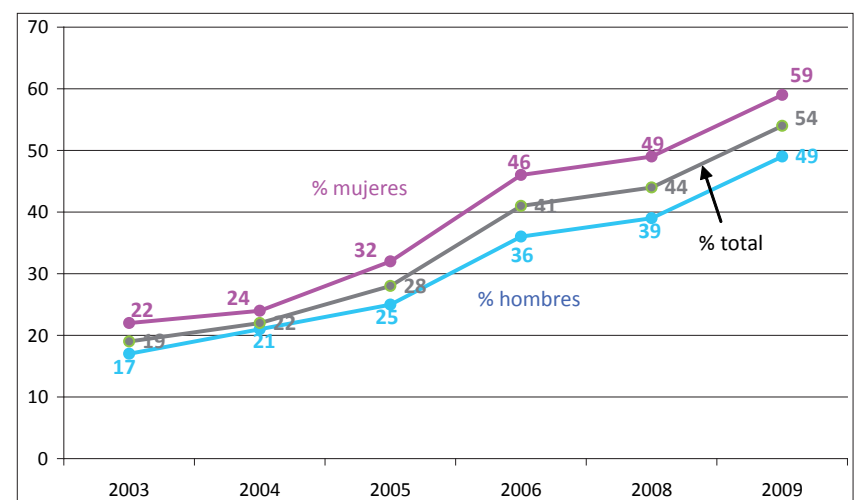

Gráfico 1. Porcentaje de personas que han utilizado internet en España para la búsqueda de información sobre temas de salud (Fuente: INE)

La encuesta del INE sobre Equipamiento y uso de tecnologías de la información y comunicación en los hogares ${ }^{9}$ (octubre de 2009) nos indica que 21 millones de personas tienen en España acceso a internet. De esta cantidad, el 54\% (12 millones de personas) la usa para la búsqueda de información sobre temas de salud. La progresión de este indicador desde el año 2003 se refleja en el gráfico 1 .

Estos datos muestran un importante crecimiento desde el año 2003. Esta progresión puede deberse en gran medida al aumento de personas que han utilizado internet en términos absolutos, pasando de 12 millones en 2003 a 21 en 2009.

En la Unión Europea también se refleja esta situación, siendo la media europea de individuos que han utilizado internet en 2004, para la búsqueda de información en salud, sobre enfermedades o nutrición, del $17 \%$, pasando al $45 \%$ en 2009 , según los datos sobre actividades de internet de usuarios de Eurostat.

Según el Segundo estudio sobre internet en España de la Fundación BBVA ${ }^{10}$ (realizado en 2008 con un tamaño muestral de 6.013 casos), el $82 \%$ de los usuarios y usuarias utilizaron la Red para la búsqueda de información, el 34,2\% de estas personas realizó búsquedas de información sobre salud y, para llegar a los sitios consultados, el $62 \%$ utilizó un buscador. Por último, el estudio muestra que los internautas conceden una nota de 7,2 puntos sobre 10 a la fiabilidad de la información consultada sobre cuestiones relacionadas con la salud.

En ocasiones, el acceso a información sobre salud a través de internet provoca en las personas usuarias un estado de desinformación, confusión, angustia, o incluso, una inclinación al autodiagnóstico y/o autotratamiento, provocando también cambios en la comunicación médico-paciente. La información en muchos casos poco fiable está suponiendo una carga adicional de trabajo para el profesional sanitario en cuanto a que es posible que se produzca una mayor exigencia o falta de confianza por parte del paciente en el profesional ${ }^{11}$.

La sociedad del conocimiento también ha traído consigo importantes cambios en la comunicación (conversación) en internet y un cambio de actitud entre todas las personas usuarias de la Red implicadas, tanto a nivel institucional como personal. Esta transformación, denominada web 2.0 o web social, se caracteriza principalmente por ser un escenario colaborativo en el que cualquier persona usuaria puede compartir, dar su opinión o contar su experiencia.

Un ejemplo de esta transformación pudo apreciarse en el terreno político en las pasadas elecciones presidenciales de los Estados Unidos en 2008, en las que el actual presidente de los Estados Unidos, Barack Obama, fichó en febrero de 2007 para su campaña presidencial, al cofundador de Facebook, Chris Hughes, como director de nuevos medios digitales, entendiendo que internet y los nuevos medios digitales eran el método de comunicación perfecto para organizar, informar y movilizar a sus seguidores.

En el entorno sanitario cada vez es más frecuente encontrar profesionales y gestores sanitarios que emplean la web 2.0 para compartir conocimiento a través de blogs ${ }^{12}$ o de otras herramientas de la web social. En el caso de los pacientes y la ciudadanía, esta nueva manera de acceder a la información sobre salud (foros, blogs, wikis, microblogging...), sin ser un indicador decisivo en la fiabilidad de la información mostrada, puede influir y ser determinante en la modificación de puntos de vista de otros consumidores de información ${ }^{13}$.

La tecnología por sí sola no será suficiente para poder resolver los problemas relacionados con el acceso a información fiable y de calidad o con el correcto funcionamiento de un sistema sanitario. Sin embargo, la adecuada gestión y desarrollo de las actuaciones a través del personal y gestores sanitarios y los profesionales de la información favorecerá la mejora de la asistencia sanitaria, dando respuesta a las necesidades y expectativas de una población dispuesta a tomar el control sobre las decisiones relacionadas con su salud ${ }^{14}$ y con un amplio acceso a información de calidad.

\section{Gestión del conocimiento en la mejora de la práctica clínica}

En el siglo XXI la competitividad de las organizaciones, de los países y de los sistemas depende de su capacidad para generar, gestionar y transformar el conocimiento en nuevos procesos, prestaciones y tecnologías al servicio de la ciudadanía ${ }^{15}$.

Se ha comprobado que compartir el mejor conocimiento disponible y fomentar el uso y constante imple- 
mentación de las tecnologías de la información son un claro beneficio para el avance de la ciencia y, en el caso de las ciencias de la salud, este uso y evolución juegan un papel cada vez más importante en la obtención de la mejor información y evidencia disponible para conseguir la excelencia en la atención, el cuidado y la seguridad de los pacientes ${ }^{16,17}$.

Las bibliotecas virtuales de salud y los repositorios de acceso abierto son dos de los escenarios ya consolidados y cuyos beneficios tienen un impacto en la mejora de la práctica clínica.

Como se recoge en un estudio ${ }^{18}$, desde la culminación de las transferencias sanitarias a finales de 2002, las diferentes Comunidades Autónomas (CCAA) han iniciado similares procesos de organización y puesta en marcha de sus propios sistemas de bibliotecas sanitarias, la mayoría de ellas creando esta estructura, que permite optimizar los medios económicos destinados a la adquisición de recursos de información bibliográfica (especialmente en formato electrónico) y servicios documentales accesibles a sus profesionales sanitarios (préstamo interbibliotecario, metabuscadores, etc.).

A pesar de que en España todavía no se ha creado una biblioteca nacional de salud, a imagen de las de Estados Unidos o Reino Unido, y con funciones similares a las de las bibliotecas nacionales del ámbito de la cultura, es importante destacar que el pasado año se puso en marcha un estudio de viabilidad para la creación de la Biblioteca Virtual del Sistema Nacional de Salud coordinado por la Biblioteca Virtual del Sistema Sanitario Público de Andalucía y en el que participan todas las CCAA.

Las diversas circunstancias y realidades de las CCAA han propiciado distintos ritmos de desarrollo de sus bibliotecas virtuales de salud (BVS). En su mayoría, éstas asumen las funciones de coordinación de las bibliotecas de los hospitales y centros sanitarios de la comunidad autónoma, proporcionando un único punto de referencia de acceso a la misma información bibliográfica para la actividad investigadora, asistencial y docente, que homogeniza los procesos y optimiza el gasto; y son la cabeza visible de la estrategia de gestión del conocimiento en el área de la salud de la Comunidad Autónoma.

Pero la diferencia del grado de avance de las BVS en las CCAA es el origen de la disparidad de acceso a una información científica de calidad por parte de los profesionales sanitarios españoles, de manera que, en función del lugar de trabajo, se tiene mayor o menor posibilidad de consultar los avances científicos.

La importancia de los repositorios de acceso abierto en una sociedad del conocimiento se ve reflejada en el retorno de la inversión efectuada, facilitando la reuti- lización de la información y el conocimiento generados por el sector público; y ha provocado la inclusión en la Ley de la ciencia, la tecnología y la innovación del artículo 35 sobre difusión en acceso abierto, que impulsará tanto la implementación de estas herramientas como el archivo en este tipo de repositorios de los resultados de la investigación financiados con fondos públicos. Una producción científica en abierto también favorece un acceso igualitario a la información de calidad con independencia del lugar de trabajo.

En la búsqueda de un sistema sanitario de excelencia centrado en mejorar la calidad de vida de la ciudadanía, el profesional de la información debe facilitar que la coordinación, la difusión y la evaluación entre el acceso al conocimiento, las TIC y los distintos actores de los procesos asistenciales estén garantizados, convirtiéndose en la herramienta dentro del sistema sanitario que lleve a cabo actuaciones destinadas a fomentar la excelencia clínica y a adoptar buenas prácticas basadas en el mejor conocimiento científico disponible.

\section{Referencias}

1. Google Groups. Afectados por la aplicación AP Madrid. http://groups.google.es/group/afectados-por-la-aplicacion-apmadrid?pli=1

2. Rivero-Corte, Pablo. "e-Salud en el Sistema Nacional de Salud". Revista eSalud.com, 2009, v. 5, n. 19.

http://www.revistaesalud.com/index.php/revistaesalud/article/view/336

3. Conferencia de alto nivel ministerial sobre sanidad digital, Barcelona, 15-18 de marzo de 2010.

http://www.ehealthweek2010.org

4. World of health IT conf and exhibiton (Wohit), Barcelona, 15-18 de marzo de 2010.

http://www.worldofhealthit.org

5. NetCraft. March 2010 Web server survey.

http://news.netcraft.com/archives/2010/03/17/march_2010_web_server_ survey.html

6. Carillo, Ana; Centor, Robert. "Physician internet medical information seeking and on-line continuing education use patterns". J contin educ health prof, 2002, v. 22, pp. 33-42.

7. Välimäki, Maritta; Nenonen, Heljä; Koivunen, Marita; Suhonen, Riita. "Patients' perceptions of internet usage and their opportunity to obtain health information". Medical informatics and the internet in medicine, 2007, v. 32, n. 4, pp. 305-14.

8. Andrews, James E.; Pearce, Kevin A.; Ireson, Carol; Love, Margaret M. "Information-seeking behaviors of practitioners in a primary care practice-based research network (PBRN)". J Med Libr Assoc, 2005, v. 93, n. 2, pp. 206-212.

9. Instituto Nacional de Estadística (INE). Encuesta sobre equipamiento y uso de tecnologías de la información y comunicación en los hogares, 2009. http://www.ine.es

10. Fundación BBVA. Segundo estudio sobre internet en España. Madrid: BBVA, 2008.

11. Ahmad, Farah; Hudak, Pamela L.; Bercovitz, Kim; Hollenberg, Elisa; Levinson, Wendy. "Are physicians ready for patients with internetbased health information?". J med internet res, 2006, v. 8, n. 3, e22.

12. Google Docs. SomosMedicina.com. Lista de blogs sanitarios en activo. http://spreadsheets. google.com/ccc?key=0AoHy-IwamTRGdHlSMWEzX2 RDWUlMVmlBRONHYTVIekE\&hl=en 
Nuestro compromiso con la mejor información disponible

www. excelenciaclinica.net

\section{Informamos y te escuchamos}

blog.plandecalidadsns. es
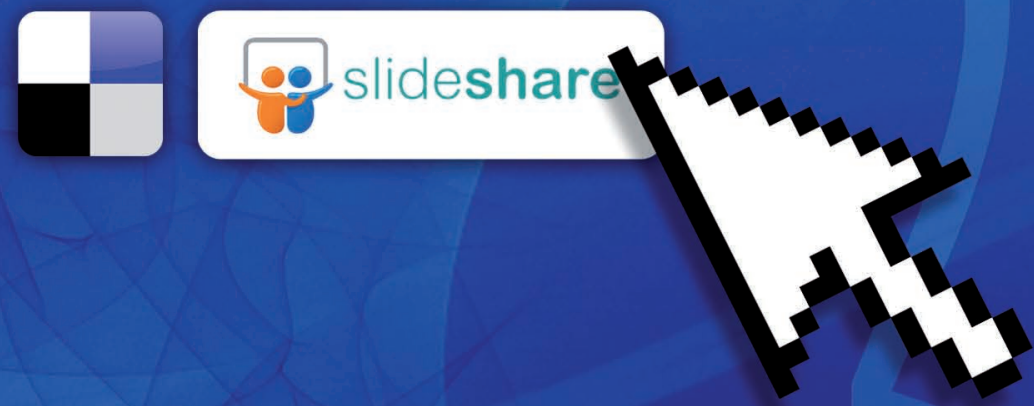